Cavendish-HEP-97/10

LPTHE-ORSAY 97/39

September 1997

hep-ph/9710333

\title{
Infrared Safe but Infinite: Soft-Gluon Divergences Inside the Physical Region"
}

\author{
S. Catani \\ INFN and Dipartimento di Fisica, Università di Firenze, \\ Largo E. Fermi 2, I-50125 Florence, Italy \\ LPTHE, Université Paris-Sud, Bâtiment 211, F-91405 Orsay, France \\ and \\ B.R. Webber \\ Cavendish Laboratory, University of Cambridge, \\ Madingley Road, Cambridge CB3 0HE, U.K.
}

\begin{abstract}
We show that QCD observables defined as infrared- and collinear-safe, according to the usual Sterman-Weinberg criteria, can nevertheless be infinite at accessible points inside phase space, to any finite order of perturbation theory. Infrared finiteness is restored after resummation of divergent terms to all orders. The resulting characteristic structure, which we call a Sudakov shoulder, represents an interesting new class of QCD predictions.
\end{abstract}

\footnotetext{
${ }^{*}$ Research supported in part by the U.K. Science and Engineering Research Council and by the EC Programme "Human Capital and Mobility", Network "Physics at High Energy Colliders", contract CHRXCT93-0357 (DG 12 COMA).
} 


\section{Introduction}

The perturbative QCD approach [1] to the calculation of hadronic cross-sections at large momentum transfer $Q$ is based on the Sterman-Weinberg criteria [2] of infrared and collinear safety, combined with the factorization theorem for mass singularities [3]. A hadronic observable is said to be infrared and collinear safe if it is insensitive to the emission of soft momenta and to the splitting of a final-state momentum into collinear momenta. The properties of infrared and collinear safety guarantee that the long-distance component of the scattering process, which is controlled by non-perturbative phenomena, is suppressed by some inverse power of $Q$ as the hard scale $Q$ increases. Thus, the observable is dominated by short distances and the short-distance component, which depends only logarithmically on $Q$, is computable as a power-series expansion in the strong coupling $\alpha_{S}(Q)$.

It is commonly assumed that the consequences of the Sterman-Weinberg criteria are even wider: the power-series in $\alpha_{S}$ is assumed to have coefficients that are finite order by order in perturbation theory. Interpreted in this way, the criteria become a statement on the infrared and collinear finiteness of the fixed-order perturbative expansion.

In this paper we would like to point out that, in general, this strong interpretation of the criteria is not valid. In fact, the perturbative expansions of infrared- and collinear-safe quantities can be divergent order by order in perturbation theory and these divergences are still produced by the radiation of soft and/or collinear partons.

The presence of logarithmic singularities that spoil the convergence of the perturbative expansion near the exclusive boundaries of the phase space of infrared- and collinear-safe observables is well known [411]. These singularities reflect the difficulty in extending the Sterman-Weinberg criteria towards extreme kinematical regions and, in many cases, they can be handled by all-order resummation methods.

The main issue to be discussed in the present paper concerns a new general class of singularities that appear inside the physical region of the phase space. They arise whenever the observable in question has a non-smooth behaviour in some order of perturbation theory at an accessible point, which we shall call a critical point, inside phase space. This can happen if the phase-space boundary for a certain number of partons lies inside that for a larger number, or if the observable itself is defined in a non-smooth way. In either case, if the distribution of the observable is actually discontinuous at that point in some order, it will become infinite there to all (finite) higher orders. If it is continuous but not smooth, i.e. if some derivatives are discontinuous, then the higher-order predictions will become unstable, with infinite derivatives, at that point.

There are several possible reactions to this state of affairs. One can try to avoid nonsmooth observables altogether. As we shall see, this actually rules out some commonly-used quantities. One can use such observables but avoid the phase-space regions near critical points. In this case, one still needs some understanding of the phenomenon in order to assess the extent of the dangerous regions. Alternatively, one can identify the problematic terms in each order of perturbation theory and resum them to all orders. As mentioned above, this approach has been used successfully for the treatment of singularities near the exclusive phase-space boundary in many observables. We advocate a similar approach to the new class of singularities discussed here, although much work remains to be done in order to reach a comparable level of understanding. As a first step in this direction, we 
carry out the resummation of leading double-logarithmic terms, and show that it leads to smooth behaviour at the critical point.

In the following Section we give a general discussion of the effects of soft and/or collinear parton emission in fixed-order QCD perturbation theory. We first review the cancellation mechanism which normally ensures the regularity of infrared- and collinear-safe observables, and the way in which this breaks down at the exclusive boundary of phase space. We then show how a similar breakdown will occur inside phase space when the observable is defined in a non-smooth way, and describe the general form of the resulting singularities.

In Sect. 3 we discuss in some detail two specific cases in which such singularities actually appear. The first is the distribution of the $C$-parameter [12,13], a well-known event shape variable for $e^{+} e^{-}$annihilation final states. The physical region is $0<C<1$, but at the point $C=3 / 4$ there is a divergence in the perturbative prediction of the $C$-distribution at second and higher orders. The second example is taken from jet physics in hadronhadron collisions, and concerns the jet shape or profile function, which describes the angular distribution of energy with respect to the jet axis [14. Here one finds a critical point at the (arbitrary) boundary of the cone used to define the jets, which leads to divergences in jet shapes and exclusive multijet cross sections at next-to-leading order and beyond.

In Sect. 1 we discuss the resummation of these divergences to all orders. We expect on general grounds that infrared finiteness will be restored by resummation, and we show that this is the case for the leading double-logarithmic terms. After resummation, one obtains a characteristic structure which is not only finite but smooth (infinitely differentiable) at the critical point. By analogy with the characteristic Sudakov peak generated by a similar mechanism near the exclusive phase space boundary, we call this structure a Sudakov shoulder. We also discuss the resummation of a more general class of non-smooth behaviours at the critical point, which give rise to different structures related to the Sudakov shoulder.

Finally in Sect. 5 we summarize our main results and conclusions.

\section{Singularities in fixed order}

The singularities we are interested in are due to the radiation of soft and/or collinear partons. In perturbative calculations, the soft and collinear regions of the phase space lead to infrared divergences in the QCD matrix elements. In the case of infrared and collinear safe observables the divergences cancel upon adding real and virtual contributions but, precisely speaking, the cancellation does not necessarily take place order by order in perturbation theory. In fact, in particular kinematic configurations real and virtual contributions can be highly unbalanced, spoiling the cancellation mechanism.

\section{Soft-gluon cancellation}

In order to discuss this point most generally, we denote by $C$ an infrared- and collinearsafe observable defined in a hard-scattering process at the scale $Q$. We can always assume that $C$ is dimensionless and varies in the kinematic range $0 \leq C \leq 1$. We denote by $\hat{\sigma}^{(n)}(C)$ a related cross section or distribution evaluated at $n$-th order in perturbation theory and

${ }^{\dagger}$ The perturbative distribution $\hat{\sigma}(C)$ can be either a measurable quantity or a short-distance coefficient function that appears in the factorization theorem. 
consider the effect of soft-gluon radiation at the next perturbative order. If $1-z$ denotes the fraction of the centre-of-mass energy involved in the radiative process, virtual $(v)$ and real $(r)$ soft gluons affect the cross section with the following emission probabilities

$$
\begin{aligned}
& \frac{d w_{v}(z)}{d z}=-\frac{\alpha_{S}(Q)}{\pi} \delta(1-z) \int_{0}^{1-\epsilon} \frac{d z^{\prime}}{1-z^{\prime}} \ln \frac{1}{1-z^{\prime}} \\
& \frac{d w_{r}(z)}{d z}=\frac{\alpha_{S}(Q)}{\pi} \frac{1}{1-z} \ln \frac{1}{1-z} \Theta(1-z-\epsilon) .
\end{aligned}
$$

The expressions (2.1,2.2) are valid to double-logarithmic accuracy and arise from the combination of the customary bremsstrahlung spectrum $d \omega / \omega$ with the angular distribution $d \theta^{2} / \theta^{2}$ for collinear radiation. Here we have introduced an unphysical cutoff $\epsilon$ on the minimum energy fraction of both gluons because the probabilities in Eqs. (2.1) and (2.2) are separately divergent if the cutoff is absent. However, in the case of infrared- and collinearsafe observables, real and virtual emissions contribute with equal weight $K$ to cross sections, although the actual value of the coefficient $K$ depends on the particular observable under studyf. Thus, adding the real and virtual terms, the physical limit $\epsilon \rightarrow 0$ can be safely taken, leading to a finite differential probability:

$$
\begin{aligned}
\frac{d w(z)}{d z} & =K \lim _{\epsilon \rightarrow 0}\left[\frac{d w_{v}(z)}{d z}+\frac{d w_{r}(z)}{d z}\right] \\
& =a\left(\frac{1}{1-z} \ln \frac{1}{1-z}\right)_{+},
\end{aligned}
$$

where $a=K \alpha_{S}(Q) / \pi$ and, as usual, the plus-prescription $[g(z)]_{+}$stands for

$$
\int_{0}^{1} d z f(z)[g(z)]_{+} \equiv \int_{0}^{1} d z[f(z)-f(1)] g(z)
$$

The plus-prescription in Eq. (2.4) is the result of the cancellation of the dependence on the unphysical cutoff $\epsilon$ and defines a well-behaved distribution, i.e. one leading to nonsingular functions, when acting on any smooth function $f(z)$ that is finite at $z=1$. However, this is the sole cancellation mechanism of infrared singularities that is guaranteed by infrared and collinear safety in fixed-order perturbation theory. The Sterman-Weinberg criteria do not imply that the coefficients of the perturbative expansion are non-singular functions. In fact, normally these coefficients are (or are obtained from) singular generalized functions or distributions, like the soft-gluon probability in Eq. (2.4). Such distributions lead to finite quantities only when they are integrated with sufficiently smooth test functions.

In particular, the $(n+1)$-st order cross section $\hat{\sigma}^{(n+1)}(C)$ is obtained by combining the soft-gluon probability with the $n$-th order term $\hat{\sigma}^{(n)}(C)$ and the latter may well not be a smooth function of $C$ for all values of $C$. In this case, the real and virtual contributions are effectively unbalanced and singularities show up in the fixed-order perturbative expansion.

In order to discuss these singularities, we have to specify how the observable $C$ depends on the soft-gluon energy. We consider for definiteness the case in which $C$ increases when

\footnotetext{
${ }^{\ddagger}$ In the case of measurable quantities, the coefficient $K$ is typically positive definite. If instead the distribution $\hat{\sigma}(C)$ is a short-distance coefficient function, the sign of $K$ depends strongly on the factorization scheme used to define the parton distribution functions.
} 
soft partons are radiated in the final state. Thus, the emission of a soft gluon with energy fraction $1-z$ produces a change $C \rightarrow C+\delta C$, where $\delta C=\mathcal{O}(1-z)$, and, without loss of generality, we can write the next perturbative order as follows:

$$
\hat{\sigma}^{(n+1)}(C)=\int_{0}^{C} d y \hat{\sigma}^{(n)}(C-y)\left(\frac{d w(z)}{d z}\right)_{z=1-y}+\ldots
$$

where the dots stand for less singular terms, if any. Because of the character of the softgluon distribution $d w(z) / d z$, if $\hat{\sigma}^{(n)}$ is not smooth at some point in the kinematic range $0 \leq C \leq 1$, the integral on the right-hand side of Eq. (2.6) can be divergent.

\section{Singularities at the exclusive boundary}

The most common and extensively studied soft-gluon singularities are those arising at an exclusive boundary of the phase space. In this kinematic regime, the radiative tail of real emission is strongly suppressed, producing the loss of balance with the virtual contribution. Since the observable $C$ that we are considering increases when soft partons are radiated, the exclusive boundary is the region $C \rightarrow 0$. Here the non-smooth behaviour of $\hat{\sigma}^{(n+1)}(C)$ is simply due to the associated phase-space constraint $\Theta(C)$. Inserting Eq. (2.4) into Eq. (2.6) we have:

$$
\hat{\sigma}^{(n+1)}(C)=a\left[-\frac{1}{2} \hat{\sigma}^{(n)}(C) \ln ^{2} C+\int_{0}^{C} \frac{d y}{y} \ln \frac{1}{y}\left(\hat{\sigma}^{(n)}(C-y)-\hat{\sigma}^{(n)}(C)\right)\right]+\ldots .
$$

Thus, even if $\hat{\sigma}^{(n)}(C)$ is finite $e^{\beta}$ as $C \rightarrow 0$, the first term in the square bracket produces a divergent contribution at the kinematical boundary:

$$
\hat{\sigma}^{(n+1)}(C) \underset{C \rightarrow 0}{\sim}-\frac{a}{2} \hat{\sigma}^{(n)}(C) \ln ^{2} C+\ldots .
$$

These double-logarithmic terms are usually called soft-gluon singularities of Sudakov type. They appear in the perturbative expansions of many $e^{+} e^{-}$shape variables in the two-jet limit [4,5]. The observable $C$, for instance, can be the $C$-parameter 12, 13 or $C=1-T$, where $T$ is the thrust [15]. Similar soft-gluon effects occur in hadron collisions for $Q_{\perp}$-distributions in the Drell-Yan process $\left(C \sim Q_{\perp} / Q\right)$ [6] and for the production of systems of high mass $M$ near threshold $(1-C \sim M / \sqrt{S}$, where $\sqrt{S}$ is the centre-ofmass energy). Outstanding examples of these systems are lepton pairs with large invariant mass produced via the Drell-Yan mechanism [7], the hadronic final state in deep-inelastic lepton-hadron scattering [8], heavy quark-antiquark pairs [9-11] and pairs of jets at large transverse momentum [11].

\section{Singularities inside the physical region}

Another possible source of soft-gluon singularities, which has received less attention in the literature, arises when $\hat{\sigma}^{(n)}(C)$ has non-smooth behaviour at a certain value $C=C_{0}$ inside the physical region of phase space. This can happen if the phase-space boundary for a certain number of partons lies inside that for a larger number, or if the observable $C$ is defined in a non-smooth way. We discuss examples of both types below.

${ }^{\S}$ If $\hat{\sigma}^{(n)}(C)$ is divergent at $C=0$, the double-logarithmic singularity in Eq. (2.8) is enhanced and can also be non-integrable. 
The diagnosis of singularities inside the physical region is physically less obvious than that for singularities at the phase-space boundary. At the exclusive boundary we are dealing with a multi-scale process: besides the hard scale $Q$, there is also another natural scale, the inelasticity scale $Q^{\prime} \sim C Q$, that plays a relevant role. The Sudakov singularities in Eq. (2.8) follow from the large mismatch between these two scales, $Q^{\prime} \ll Q$ when $C \rightarrow 0$. For singularities inside the physical region, on the other hand, the lack of balance between real and virtual contributions is just produced by the sharpness of the distribution around the critical point $C_{0}$, and the actual identification of a critical point requires careful analysis of the kinematics and dynamics.

To show how these singularities arise, we denote the cross section for $C<C_{0}$ by $\hat{\sigma}_{-}(C)$ and that for $C>C_{0}$ by $\hat{\sigma}_{+}(C)$, and we can suppose that at the $n$-th order both $\hat{\sigma}_{-}^{(n)}$ and $\hat{\sigma}_{+}^{(n)}$ are infinitely differentiable functions. Then we find from Eq. (2.7) that $\hat{\sigma}_{-}^{(n+1)}(C)$ is infinitely differentiable and regular at $C=C_{0}$ while $\hat{\sigma}_{+}^{(n+1)}(C)$ can be singular there. The difference comes from the second term in the square bracket of Eq. (2.7) and is given by

$$
\begin{aligned}
\hat{\sigma}_{+}^{(n+1)}(C)-\hat{\sigma}_{-}^{(n+1)}\left(C_{0}\right) & =-\frac{a}{2} \ln ^{2}\left(C-C_{0}\right)\left\{\left[\hat{\sigma}_{+}^{(n)}\left(C_{0}\right)-\hat{\sigma}_{-}^{(n)}\left(C_{0}\right)\right]\right. \\
& \left.+\left(C-C_{0}\right)\left[\hat{\sigma}_{+}^{(n) \prime}\left(C_{0}\right)-\hat{\sigma}_{-}^{(n) \prime}\left(C_{0}\right)\right]+\ldots\right\}+\ldots,
\end{aligned}
$$

where $\hat{\sigma}_{-}^{(n) \prime}(C)$ denotes the first derivative of the distribution with respect to $C$ and the dots indicate terms that are less singular as $C \rightarrow C_{0}$. Thus if $\hat{\sigma}_{+}^{(n)}\left(C_{0}\right) \neq \hat{\sigma}_{-}^{(n)}\left(C_{0}\right)$, i.e. $\hat{\sigma}^{(n)}(C)$ has a step at $C=C_{0}$, then $\hat{\sigma}_{+}^{(n+1)}(C)$ has a double-logarithmic divergence at that point. The same mechanism leading to Eq. (2.9) will enhance the double-logarithmic singularity by further integer powers of $\ln \left(C-C_{0}\right)$ in yet higher orders 7 .

In general, a step at some point $C=C_{0}$ inside the physical region at the $n$-th order of perturbation theory always generates more and more divergent contributions at $C=C_{0}$ in higher and higher orders. To any fixed order $m>n$, the cross section $\hat{\sigma}_{+}^{(m)}\left(C_{0}\right)$ is infinite, because the emission of arbitrarily soft gluons is not cancelled by virtual corrections at that point. One may regard this as a failure of the Sterman-Weinberg criteria for infrared and collinear finiteness in fixed-order perturbation theory: we have considered an observable which satisfies the criteria, but nevertheless the perturbative prediction at a point inside the physical phase-space is divergent.

One might think that the construction of an infrared and collinear safe quantity, which at the $n$-th perturbative order has a step-like behaviour inside the physical region, is quite abstract. In fact, this can happen if, due to the finite number of partons involved in the calculation at that order, they can occupy the phase-space region only up to a value $C=C_{0}$ that is smaller than the maximum value permitted by kinematics. To produce a step it is sufficient that the computed distribution does not vanish at $C=C_{0}$. As we shall see in Sect. 8 , the distribution of the $C$-parameter in $e^{+} e^{-}$annihilation has these features.

A similar behaviour can occur if the infrared and collinear safe observable $C$ is used to classify the topology of hadronic events. In this case sharp structures can easily be produced. For instance, $C$ can be a jet resolution parameter that is defined in a nonsmooth way (e.g. it can have a non-trivial and non-smooth dependence on energies and angles of partons). Then the topology of the (partonic) event, e.g. the number of jets,

\footnotetext{
『Note that all these singularities are integrable in a neighbourhood of the critical point.
} 
can suddenly change at a certain value $C_{0}$. At that point the rate $\hat{\sigma}^{(n)}(C)$ for producing a fixed number of jets can have a step. Moreover, a corresponding step, with the opposite discontinuity, will appear in the production rate relating to a different number of jets. At the next perturbative order, the step in $\hat{\sigma}^{(n)}(C)$ leads to a singularity for $C>C_{0}$ and, by the same argument as in Eq. (2.9), the step in the other jet rate leads to a similar singularity for $C<C_{0}$. Since the sum of the two jet rates remains finite, both of them will show a logarithmic divergence on both sides of the step, that is, above and below the critical point $C=C_{0}$. This may appear in contradiction with the statement above Eq. (2.9). However, since we are considering a variable $C$ that is defined in a non-smooth way, it is not monotonic with respect to soft-gluon energies. Therefore, the simple formula in Eq. (2.6) cannot be applied, although the general relation between steps and singularities is still valid. A phenomenologically-relevant example of this kind of singularity is also considered in Sect. 3 .

The result in Eq. (2.9) also allows us to discuss the effect of soft-gluon radiation on nonsmooth quantities when they do not have a step-like behaviour. For example, the $n$-th-order distribution $\hat{\sigma}^{(n)}(C)$ may be continuous but not its first derivative $\left(\hat{\sigma}_{+}^{(n) \prime}\left(C_{0}\right) \neq \hat{\sigma}_{-}^{(n) \prime}\left(C_{0}\right)\right)$, so that $\hat{\sigma}^{(n)}(C)$ has a sharp edge at $C=C_{0}$. An example would be $C=1-T$ where $T$ is the $e^{+} e^{-}$thrust variable, which has kinematic range $\frac{1}{2} \leq T \leq 1$ but is zero in first order $(n=1)$ for $T<\frac{2}{3}$ and vanishes linearly as $T \rightarrow T_{0}=\frac{2}{3}^{+}$. Then $\hat{\sigma}^{(n+1)}(C)$ is also continuous but has a cusp at $C=C_{0}$, where $\hat{\sigma}_{+}^{(n+1) \prime}(C)$ diverges double-logarithmically of $C=1-T$, from Eq. (2.9) we obtain that $\hat{\sigma}_{+}^{(2) \prime}(C)$ diverges to negative infinity" at $C_{0}=1-T_{0}=\frac{1}{3}$. In higher orders $\hat{\sigma}(C)$ remains continuous but the degree of divergence of its derivative is enhanced by an increasing number of double logs, so the edge gets more and more like a step order by order in perturbation theory.

An edge at a certain order thus does not produce divergences in the perturbative expansion. Nonetheless, fixed-order predictions are likely to be unreliable near the critical point because the shape of the distribution, independently of its magnitude, is highly unstable with respect to radiative corrections. For instance, if $\hat{\sigma}^{(n)}(C)$ is decreasing at the edge and the perturbative coefficient $a$ in Eq. (2.9) is positive, the cusp at the next order leads to an increasing distribution for $C \geq C_{0}$.

\section{$3 \quad$ Examples}

Here we illustrate the above general discussion with two specific examples of distributions of well-known observables which do develop divergences at points inside the physical phase space, due to imperfect cancellation between real and virtual soft-gluon singularities.

\subsection{The $C$-parameter in $e^{+} e^{-}$annihilation}

The $C$-parameter is an infrared- and collinear-safe observable that was introduced in Refs. [12 to describe the shape of hadronic events produced by $e^{+} e^{-}$annihilation. De-

\footnotetext{
"In general, if $\hat{\sigma}^{(n)}$ is continuous up to its $k$-th derivative, the double-logarithmic singularity affects the $k$-th derivative of $\hat{\sigma}^{(n+1)}$.

** This cusp is visible in Fig. 1 of Ref. [16].
} 
noting by $\boldsymbol{p}_{i}^{\alpha}$ the components of the three-momentum of any final-state particle $i$, one first considers the linearized momentum tensor

$$
\Theta^{\alpha \beta}=\frac{\sum_{i} \boldsymbol{p}_{i}^{\alpha} \boldsymbol{p}_{i}^{\beta} /\left|\boldsymbol{p}_{i}\right|}{\sum_{j}\left|\boldsymbol{p}_{j}\right|},
$$

and computes its eigenvalues $\lambda_{i}$. The $C$-parameter is then defined as follows

$$
C=3\left(\lambda_{1} \lambda_{2}+\lambda_{2} \lambda_{3}+\lambda_{3} \lambda_{1}\right)
$$

By definition the eigenvalues satisfy the following constraints

$$
0 \leq \lambda_{i} \leq 1, \quad \sum_{i} \lambda_{i}=1
$$

so that the $C$-parameter varies in the kinematic range $0 \leq C \leq 1$. In particular, $C=0$ for a perfectly two-jet-like final state (e.g. $\lambda_{2}=\lambda_{3}=0, \lambda_{1}=1$ ) and $C=1$ for an isotropic and acoplanar distribution of final-state momenta $\left(\lambda_{1}=\lambda_{2}=\lambda_{3}=\frac{1}{3}\right)$.

In fact, the maximal value $C=1$ can only be achieved when there are four or more final-state particles. Planar events have one vanishing eigenvalue and occupy the kinematic region $C \leq \frac{3}{4}$. Hence, we are in a situation where the phase-space boundary for three particles is below the kinematic limit of the shape variable. For a general three-particle state

$$
C=C_{3}\left(x_{1}, x_{2}\right)=6\left(1-x_{1}\right)\left(1-x_{2}\right)\left(1-x_{3}\right) /\left(x_{1} x_{2} x_{3}\right)
$$

where $x_{i}=2 p_{i} \cdot Q / Q^{2}$ are the centre-of-mass energy fractions $\left(x_{1}+x_{2}+x_{3}=2\right)$. The maximum value $C=\frac{3}{4}$ for three particles corresponds to the symmetric configuration $x_{1}=x_{2}=x_{3}=\frac{2}{3}$.

In perturbation theory, the distribution of the $C$-parameter for $C \neq 0$ has the general form

$$
\frac{1}{\sigma_{0}} \frac{d \sigma}{d C}=\bar{\alpha}_{S} A(C)+\bar{\alpha}_{S}^{2} B(C)+\mathcal{O}\left(\alpha_{S}^{3}\right)
$$

where $\bar{\alpha}_{S}=\alpha_{S}(Q) / 2 \pi$ and we normalize to the Born cross section $\sigma_{0}$, as was done in Ref. [17]. The two functions $A(C)$ and $B(C)$ can be identified respectively with the distributions $\hat{\sigma}^{(n)}$ and $\hat{\sigma}^{(n+1)}$ of Sect. 2. In particular, we are exactly in the situation where Eq. (2.6) applies because, as can be checked easily, the $C$-parameter increases when additional soft particles are produced.

The first-order distribution $A(C)$ is given by the $q \bar{q} g$ final state:

$$
A(C)=\int_{0}^{1} d x_{1} d x_{2} \Theta\left(x_{1}+x_{2}-1\right) M\left(x_{1}, x_{2}\right) \delta\left[C-C_{3}\left(x_{1}, x_{2}\right)\right]
$$

where

$$
M\left(x_{1}, x_{2}\right)=C_{F} \frac{x_{1}^{2}+x_{2}^{2}}{\left(1-x_{1}\right)\left(1-x_{2}\right)} .
$$

Note that the matrix element (3.7) has a finite value $M_{0}=M\left(x_{1}=x_{2}=\frac{2}{3}\right)=8 C_{F}$ in the symmetric configuration where $C=\frac{3}{4}$. Furthermore one finds that the available phasespace remains finite as $C \rightarrow \frac{3}{4}^{-}$. Therefore, the $\mathcal{O}\left(\alpha_{S}\right)$ distribution (3.6) has a step at

${ }^{\dagger}$ Note that, consistently with the general discussion in Sect. 2, the first-order distribution $A(C)$ in Eq. (3.6) diverges at the exclusive boundary $C=0$ of the phase space. This divergence and the corresponding ones in higher orders can be resummed [13] using the techniques of Ref. «4. 


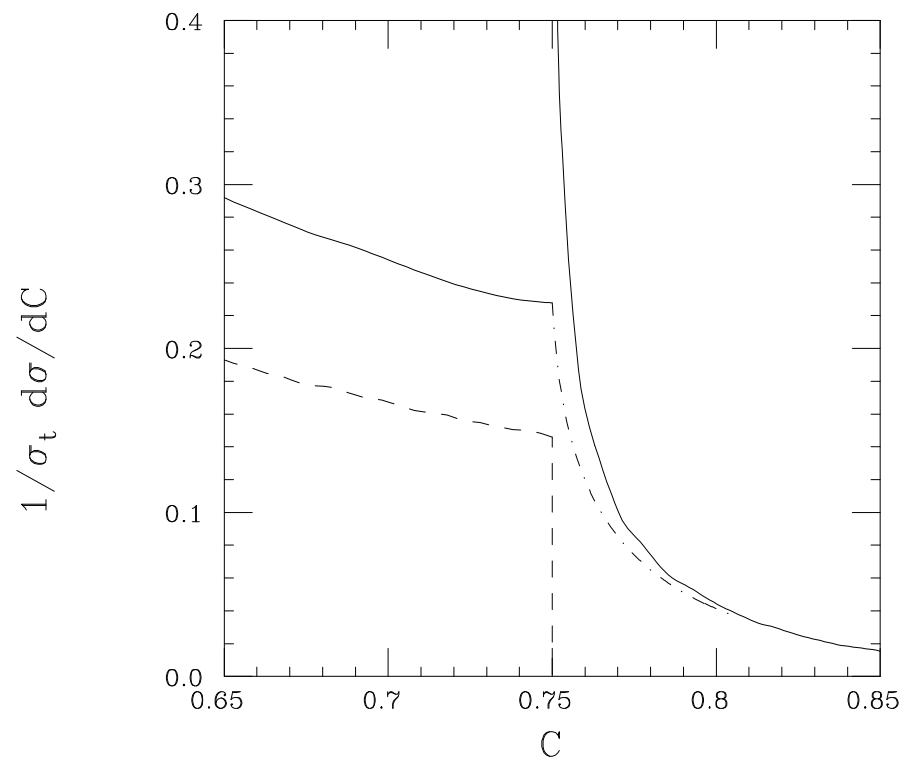

Figure 1: Predictions of the $C$-parameter distribution for $\alpha_{S}=0.12$. Dashed: $\mathcal{O}\left(\alpha_{S}\right)$. Solid: $\mathcal{O}\left(\alpha_{S}^{2}\right)$. Dot-dashed: resummed.

the three-parton upper limit $C=\frac{3}{4}$ :

$$
\begin{aligned}
& A(C) \underset{C \sim 3 / 4}{\simeq} A\left(\frac{3}{4}\right) \Theta\left(\frac{3}{4}-C\right), \\
& A\left(\frac{3}{4}\right)=\frac{32}{243} \pi \sqrt{3} M_{0}=\frac{256}{243} \pi \sqrt{3} C_{F} .
\end{aligned}
$$

The derivative of $A(C)$ is also finite at $C=\frac{3}{4}^{-}$: in fact

$$
A^{\prime}\left(\frac{3}{4}\right)=-\frac{8}{3} A\left(\frac{3}{4}\right) \text {. }
$$

The resulting first-order prediction in the vicinity of $C=\frac{3}{4}$ is shown by the dashed curve in Fig. 1.

According to Sect. 2, the second-order contribution $B(C)$ should be smooth as $C \rightarrow \frac{3}{4}^{-}$ but should have a double-logarithmic singularity as $C \rightarrow \frac{3}{4}^{+}$. This is indeed the case, as shown by the solid curve in Fig. 1. Denoting $B(C)$ above/below $C=\frac{3}{4}$ by $B_{ \pm}(C)$ we find using the Monte Carlo matrix element evaluation program EVENT [17]

$$
B_{-}\left(\frac{3}{4}\right)=230 \pm 10 .
$$

Performing the analytic calculation for $C \rightarrow \frac{3}{4}^{+}$, we find

$$
B_{+}(C) \simeq A\left(\frac{3}{4}\right)\left[\left(2 C_{F}+C_{A}\right)(1-\delta) \ln ^{2} \delta+\left(3 C_{F}+\frac{11}{6} C_{A}-\frac{1}{3} N_{f}\right) \ln \delta\right]+h(C)
$$

where

$$
\delta=\frac{8}{3}\left(C-\frac{3}{4}\right)
$$

and the remainder is expected to take the form

$$
h(C)=h\left(\frac{3}{4}\right)+\mathcal{O}(\delta \ln \delta) .
$$




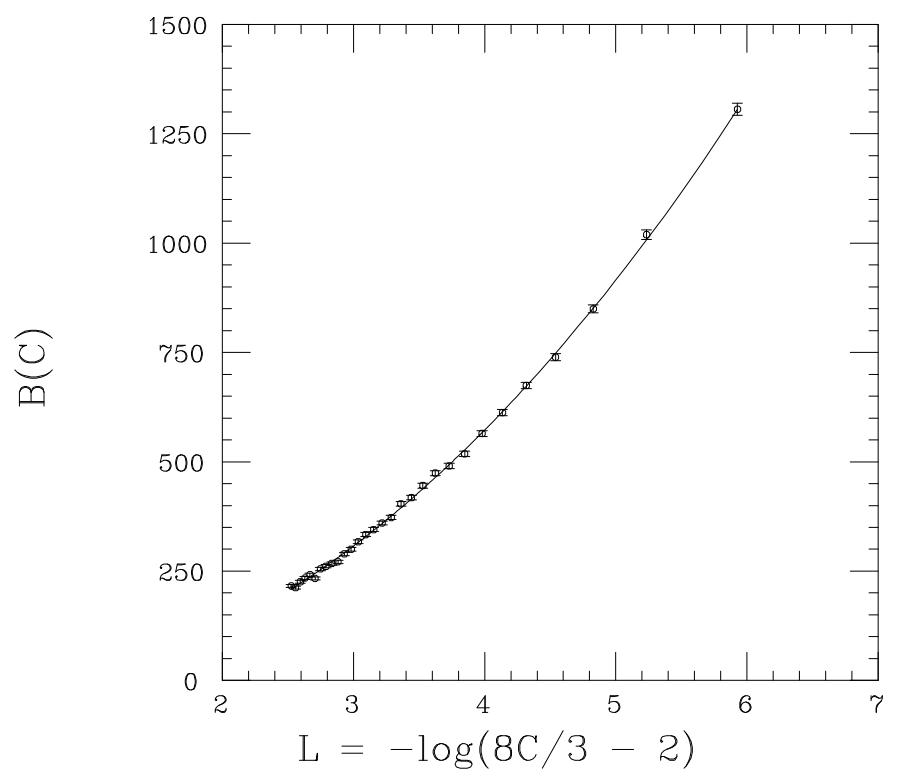

Figure 2: Second-order prediction of the $C$-parameter distribution for $C \rightarrow \frac{3}{4}^{+}$. Points: EvENT Monte Carlo. Curve: Eqs. (3.12 3.15).

Using Eq. (3.10), we see that the first term in the square bracket on the right-hand side of Eq. (3.12) is in complete agreement with Eq. (2.9) with $a=\left(2 C_{F}+C_{A}\right) \alpha_{S}(Q) / \pi$. The second term in the square bracket is due to collinear but non-soft parton splitting: singlelogarithmic contributions of this type were neglected in the simplified discussion of Sect. 2 , which was valid to double-logarithmic (DL) accuracy only.

Comparing our analytic calculation with numerical data from EVENT [17], we find good agreement, as shown in Fig. 2. The value of the constant $h\left(\frac{3}{4}\right)$ in Eq. (3.14) is found to be

$$
h\left(\frac{3}{4}\right)=146 \pm 3 .
$$

\subsection{Jet shapes in hadron collisions}

As our second example we study the angular distribution of transverse energy $\left(E_{T}\right)$ in jets produced at high $E_{T}$ in hadron-hadron collisions [14. Here it is customary to define a jet by a cone algorithm [18], that is, by maximizing $E_{T}$ with respect to the direction of a cone of opening angle $R$ in pseudorapidity, azimuth $(\eta, \phi)$ space. The cone axis then defines the jet axis $\left(\eta_{J}, \phi_{J}\right)$. Alternatively a $k_{\perp}$-clustering algorithm can be used [19]. In this case again a parameter $R$ can be introduced, effectively representing the jet cone size [20].

Several different versions of the cone algorithm are actually used both in theoretical calculations and in experimental analyses. They differ with regard to minor details and sometimes major points. The latter are related to infrared and collinear safety. In some versions [21], the direction of the unassigned cluster (parton) with the highest $E_{T}$ is used to start the clustering procedure. Since the highest- $E_{T}$ parton is not stable with respect to collinear splitting, these algorithms are collinear unsafe. Their unsafeness certainly affects any perturbative calculation that involves more than 5 partons in the final state. Collinear safety is fulfilled by iterative-cone algorithms that use all clusters, possibly excluding those with $E_{T}$ below a fixed threshold value [22], as seed directions of the cone. Nonetheless, these 
algorithms are still infrared unsafe in the absence of an $E_{T}$-threshold [23], and therefore strongly affected by the threshold when one is applied [24]. In iterative-cone algorithms, infrared and collinear safety can be achieved [25] by using the $E_{T^{-}}$weighted midpoints of all pairs of jets (partons) as additional seed directions. In the following, by 'cone algorithm' we refer to these improved versions.

We consider here the differential jet shape or profile function $\psi(r)$, defined such that $\psi(r) d r$ is the fraction of transverse energy lying within a ring of radius $r$, width $d r$, centred on the jet axis. We have

$$
\psi(r)=\frac{\sum_{i} E_{T i} \delta\left(r-R_{i J}\right)}{\sum_{k} E_{T k} \Theta\left(R-R_{k J}\right)}
$$

where

$$
R_{i J} \equiv \sqrt{\left(\eta_{i}-\eta_{J}\right)^{2}+\left(\phi_{i}-\phi_{J}\right)^{2}}
$$

and the sums over $i, k$ can be over either all particles or only those particles assigned to the jet. The function $\psi(r)$ has been studied in detail in Ref. [24, and many of the points we make are also discussed there.

For $r>0, \psi(r)$ starts at order $\alpha_{S}$ and at this order its $r$-dependence is due to 3-parton final-states. Let us simplify the discussion by considering a cone size $R<\pi / 3$ and three partons of momenta $p_{1}, p_{2}, p_{3}$ with transverse-energy ordering, e.g. $E_{T 1}>E_{T 2}>E_{T 3}$. In this case there are two possible jet configurations:

a) jets $=\{1\},\{2\},\{3\} \quad$ if $\quad R_{23}^{\text {alg }}>R$

b) jets $=\{1\},\{23\} \quad$ if $R_{23}^{\text {alg }}<R$

where $R_{23}^{a l g}$ depends on the jet-algorithm:

$$
R_{23}^{\text {cone }}=\frac{E_{T 2}}{E_{T 2}+E_{T 3}} R_{23}, \quad R_{23}^{k_{\perp}}=R_{23}
$$

The jet shape $\psi(r)$ can be written in terms of two contributions $\psi_{\text {in }}(r)$ and $\psi_{\text {out }}(r)$, arising from particles assigned and not assigned to the jet, respectively. To order $\alpha_{S}$, the two contributions are proportional to the following expressions

$$
\begin{aligned}
\psi_{\text {in }}^{(1)}(r) \propto & \Theta\left(R-R_{23}^{\text {alg }}\right) \delta(J-\{23\}) \\
\cdot & {\left[E_{T 2} \delta\left(r-\frac{E_{T 3}}{E_{T 2}+E_{T 3}} R_{23}\right)+E_{T 3} \delta\left(r-\frac{E_{T 2}}{E_{T 2}+E_{T 3}} R_{23}\right)\right], } \\
\psi_{\text {out }}^{(1)}(r) \propto & \Theta\left(R_{23}^{\text {alg }}-R\right)\left[\delta(J-\{2\}) E_{T 3}+\delta(J-\{3\}) E_{T 2}\right] \delta\left(r-R_{23}\right),
\end{aligned}
$$

where we use the notation

$$
\delta(J-\{2\}) \equiv \delta\left(E_{T J}-E_{T 2}\right) \delta\left(\eta_{J}-\eta_{2}\right) \delta\left(\phi_{J}-\phi_{2}\right)
$$

and likewise for $\delta(J-\{3\})$ and $\delta(J-\{23\})$. Equations (3.19) and (3.20) show that $\psi_{\text {in }}^{(1)}(r)$ and $\psi_{\text {out }}^{(1)}(r)$ are respectively proportional to $\Theta(R-r)$ and $\Theta(r-R)$, and when $r \rightarrow R$ we have

$$
\begin{array}{lll}
\psi_{\text {in }}^{(1)}(r) \rightarrow \psi_{\text {in }}^{(1)}(R)>0, & \psi_{\text {out }}^{(1)}(r) \rightarrow 0, & \text { (cone alg. }) \\
\psi_{\text {in }}^{(1)}(r) \rightarrow 0, & \psi_{\text {out }}^{(1)}(r) \rightarrow \psi_{\text {out }}^{(1)}(R)>0 & \left(k_{\perp} \text { alg. }\right)
\end{array}
$$


In conclusion, $\psi^{(1)}(r)$ in the cone algorithm has a step at $r=R$, while in the $k_{\perp}$ algorithm it has an edge (step) if only particles assigned to the jet (all particles) are considered.

Let us now investigate possible singularities at the next order. Since $\psi^{(2)}(r) \propto \sum_{i} E_{T i}$ $\delta\left(r-R_{i J}\right)$, we should consider how the angular distance between the jet direction and that of parton $i$ is affected by collinear splitting and soft gluon radiation. In the case of collinear splitting the angular distance does not change. Thus we have to examine soft-gluon radiation with energy fraction $1-z$ and single-logarithmic emission probability $\frac{d w(z)}{d z}=a\left(\frac{1}{1-z}\right)_{+}$.

In the cone algorithm, $R_{i J}$ is always smaller than $R$ if $i$ belongs to the jet and always larger than $R$ if it does not. If $i$ belongs to the jet and a soft gluon is emitted inside the cone, cancellation of real and virtual divergences will occur as usual, leading to a smooth contribution to $\psi_{\text {in }}^{(2)}$. However, if the gluon is emitted outside the cone there is a mismatch of real and virtual contributions, which will generate a divergence at the boundary of the cone. The corresponding change in $R_{i J}$ is $-c(1-z)$, where $c>0$ depends on the kinematics. Similarly, if parton $i$ is outside the jet and a gluon is emitted into the cone, the shift in $R_{i J}$ is $+c(1-z)$. Thus, according to our general discussion in Sect. 2, we find

$$
\begin{aligned}
& \psi_{\text {in }}^{(2)}(r) \simeq \Theta(R-r)\left\{-a \psi_{\text {in }}^{(1)}(R) \ln \frac{1}{R-r}+\text { const. }\right\} \\
& \psi_{\text {out }}^{(2)}(r) \simeq \Theta(r-R) \text { const. }
\end{aligned}
$$

The coefficient $a$ is likely to be positive in most jet configurations so that $\psi^{(2)}(r)$ diverges to $-\infty$ when $r \rightarrow R$ from below and stays finite when $r \rightarrow R$ from above.

In the $k_{\perp}$ algorithm at higher orders, because of the iterative recombination procedure, $R_{i J}$ can be either larger or smaller than $R$, independently of whether $i$ is or is not a particle in the jet. Thus both $\psi_{\text {in }}^{(2)}(r)$ and $\psi_{\text {out }}^{(2)}(r)$ are non-vanishing in a neighbourhood of $r=R$. Moreover, $R_{i J}$ does not vary monotonically with $z$ when a soft gluon is added to the jet. Thus we find that $\psi_{\text {in }}^{(2)}(r)$ has a cusp at $r=R$ and $\psi_{\text {out }}^{(2)}(r)$ has the following double-sided singular behaviour

$$
\psi_{\text {out }}^{(2)}(r) \simeq a\left\{\Theta(R-r) \psi_{\text {out }}^{(1)}(R) \ln \frac{1}{R-r}-\Theta(r-R) \psi_{\text {out }}^{(1)}(R) \ln \frac{1}{r-R}+\text { const. }\right\} .
$$

The double-sided singularity follows from our general discussion in Sect. 2 on non-monotonic observables with stepwise behaviour at lower order.

Note that the singularities in Eqs. (3.24) and (3.25) are both single-logarithmic. This is because they are due to soft but non-collinear radiation.

Related problems connected with the calculation of jet cross sections in higher orders have been discussed in Ref. [23]. The definitions of the fixed-cone and iterative-cone algorithms given there are actually unsafe beyond a certain order. More precisely, since the highest unassigned $E_{T}$-cluster (parton) is used to start the clustering procedure, these algorithms are collinear unsafe. For a cone size $R<\pi / 3$, their unsafeness affects perturbative calculations that involves more than 5 final-state partons. Thus one may still perform calculations up to order $\alpha_{S}^{4}$ using these definitions, provided that divergences at critical points are carefully handled.

\footnotetext{
${ }^{\ddagger \ddagger}$ Assuming $a>0$, this agrees with the results of Ref. [24].
} 
Consider the 3-jet cross section in the iterative cone algorithm as defined in Ref. [23] at a fixed value of $R_{23}$, the angular distance between the two less energetic jets. At LO this cross section has a step at the critical point $R_{23}=R$, the cone radius. Actually it vanishes for $R_{23}<R$ and is finite and not vanishing for $R_{23}>R$. At NLO the emission of a soft gluon at a large angle produces a single-logarithmic divergence (the soft gluon not being collinear) at $R_{23}=R$, of the type discussed above.

This divergence is integrable and it does not produce any singularity in the cross section integrated over $R_{23}$. On the other hand, if the phase-space slicing method [26] is used to perform the calculation, the integrable divergence can give rise to a $\ln s_{\text {min }}$ dependence, where $s_{\min }$ is the 'slicing parameter' used for the numerical evaluation of the cross section. Such a dependence can be numerically important, even though it should disappear for sufficiently small values of $s_{\min }$.

To illustrate this point we note that the cutoff $\epsilon$ introduced in Sect. 2 to define the plus-distribution is somewhat analogous to a slicing parameter. In the case of a stepwise behaviour of $\hat{\sigma}^{(n)}$ at $C=C_{0}$, the integral up to $C_{1}>C_{0}$,

$$
\lim _{\epsilon \rightarrow 0} \int_{C_{0}}^{C_{1}} d C \hat{\sigma}^{(n+1)}(C ; \epsilon)
$$

is in fact finite, but if we keep $\epsilon$ fixed we get for $C_{1}-C_{0}<\epsilon$

$$
\int_{C_{0}}^{C_{1}} d C \hat{\sigma}^{(n+1)}(C ; \epsilon)=a\left[-\frac{1}{2} \ln ^{2} \epsilon \int_{C_{0}}^{C_{1}} d C \hat{\sigma}_{+}^{(n)}(C)+\text { const. }+\mathcal{O}(\epsilon \ln \epsilon)\right],
$$

which shows an unphysical dependence on $\ln \epsilon$. The unphysical logarithmic dependence does not cancel as long as $\epsilon$ is not sufficiently small.

\section{Resummation and the Sudakov shoulder}

Although we have shown that divergent fixed-order predictions will generally arise at nonsmooth points inside the physical region, one might feel that this is a purely academic difficulty, since the divergence at such a point $C=C_{0}$ is integrable, as long as $\hat{\sigma}^{(n)}(C)$ itself is integrable in a neighbourhood of that point. The cancellation of real and virtual divergences will therefore take place in the presence of smearing in $C$, as would be expected from non-perturbative (hadronization) effects. However, the resulting prediction will be highly unstable with respect to the order at which we stop the perturbative calculation and the amount of smearing introduced.

In particular, since the hadronization smearing should cancel divergent terms proportional to some power of $\alpha_{S}(Q)$, this would imply that non-perturbative effects scale logarithmically with $Q$, thus spoiling not only the finiteness but also the safety of the Sterman-Weinberg criteria. We should then be forced to conclude that some infrared- and collinear-safe observables are affected by non-perturbative contributions that are not power suppressed.

Alternatively, one might think that the divergence in the fixed-order prediction can be cancelled by the resummation of still higher-order perturbative corrections. One might argue that the resummation of real and virtual terms produces a Sudakov suppression of 
the divergence, similarly to the Sudakov suppression of many observables at the exclusive boundary of the phase space [46]. This scenario would lead to a resummed prediction that, at the critical point $C=C_{0}$, is finite but still has a stepwise behaviour. On physical grounds we do not anticipate this behaviour, and we should expect non-perturbative effects to fill up the gap between the two sides of the step. Since the gap is produced in perturbation theory, in this case also the necessary non-perturbative contributions could not be power suppressed.

We believe that instead the problem of divergences inside the physical region has a satisfactory solution entirely within the context of perturbation theory. Namely, the resummation of the soft-gluon contributions to all orders, rather than producing a Sudakov suppression of the divergences, leads to a structure that is continuous and smooth, indeed infinitely differentiable, at $C=C_{0}$. We call this structure a Sudakov shoulder. The existence of a Sudakov shoulder implies the restoration of validity of the Sterman-Weinberg criteria at infinite order in perturbation theory.

To demonstrate this point we first recall how soft-gluon resummation produces the Sudakov form factor at the exclusive boundary of the phase space. We thus consider the case in which $\hat{\sigma}(C)$ is a measurable quantity (with a positive soft-gluon coefficient $a$ ) and proceed to the iterative application of Eq. (2.7), retaining for simplicity only the doublelogarithmic (DL) contribution at each order. Taking into account the symmetrization with respect to exchange of soft gluons, we obtain an exponential series that, after summation in the neighborhood of the point $C=0$, gives

$$
\hat{\sigma}^{(\infty)}(C) \underset{C \rightarrow 0}{=} \exp \left\{-\frac{a}{2} \ln ^{2} C\right\} \hat{\sigma}^{\left(n_{0}\right)}(C)
$$

where $n_{0}$ represents the lowest perturbative order for our observable. The exponential term on the right-hand side of Eq. (4.1) is the customary Sudakov form factor, which suppresses the observable at the exclusive boundary.

The analogous iterative procedure in the region $C \sim C_{0}$ also leads to an exponential series which can be summed to give

$$
\hat{\sigma}_{+}^{(\infty)}(C)-\hat{\sigma}_{-}^{(\infty)}\left(C_{0}\right)=\exp \left\{-\frac{a}{2} \ln ^{2}\left(C-C_{0}\right)\right\}\left[\hat{\sigma}_{+}^{\left(n_{0}\right)}\left(C_{0}\right)-\hat{\sigma}_{-}^{\left(n_{0}\right)}\left(C_{0}\right)\right]
$$

where $n_{0}$ represents the order at which the step at $C=C_{0}$ first appears. Now we may assume that $\hat{\sigma}_{-}^{(\infty)}\left(C_{0}\right)$ has no soft-gluon singularities, since by hypothesis $C$ is always increased by soft gluon emission. It then follows from Eq. (4.2) that after resummation the cross section is finite, continuous and infinitely differentiable at $C=C_{0}$. Rather than suppressing the divergence at $C>C_{0}$, Sudakov resummation leads to the suppression of the step. The general form of the resummed cross section will be a smooth extrapolation from $\hat{\sigma}_{-}^{(\infty)}\left(C_{0}\right)$ into the region $C>C_{0}$, joining smoothly with the different asymptotic behaviour of $\hat{\sigma}_{+}^{(\infty)}(C)$ well above $C=C_{0}$. This is the characteristic structure we call a Sudakov shoulder.

The dot-dashed curve in Fig. 1 illustrates the Sudakov shoulder in the case of the $C$ parameter distribution. Here we simplify the treatment of the region $C>\frac{3}{4}$ not only by using the DL approximation for the form factor but also by approximating the lowest-order 
contribution $A(C)$ in Eq. (3.6) by a step function, $A(C) \simeq A\left(\frac{3}{4}\right) \Theta\left(\frac{3}{4}-C\right)$. We then obtain

$$
\frac{1}{\sigma} \frac{d \sigma}{d C} \simeq \bar{\alpha}_{S} A\left(\frac{3}{4}\right)\left\{\Theta\left(\frac{3}{4}-C\right)+\Theta\left(C-\frac{3}{4}\right)\left(1-\exp \left[-2 A^{(1)} \bar{\alpha}_{S} \ln ^{2}\left(C-\frac{3}{4}\right)\right]\right)\right\} \quad \text { (DL) }
$$

where

$$
A^{(1)}=C_{F}+\frac{1}{2} C_{A} .
$$

Expanding the right-hand side of Eq. (4.3) perturbatively, one finds a series of logarithmically divergent (although integrable) terms for $C \rightarrow \frac{3}{4}^{+}$. The summation of these terms to all orders leads to a finite result and the lowest-order step-like behaviour is smoothed. The resummed expression (4.3) shows a shoulder that extends beyond the value $C=\frac{3}{4}$. The shoulder becomes less steep with increasing $A^{(1)}$ or $\alpha_{S}$. Since $A^{(1)}=C_{F}+C_{A} / 2 \sim 2 C_{F}$, in an abelian theory (i.e., setting $C_{A}=0$ ) the DL shoulder would be twice as steep as in QCD. The additional broadening in the QCD case is due to the fragmentation of the gluon jet. Note that the shoulder is nevertheless still quite sharp on the scale shown in Fig. 1.

Soft-gluon resummation will smooth out perturbative predictions of infrared- and collinearsafe observables at other types of critical points inside phase space. After the case of a step, the next simplest example is that of a critical point $C=C_{0}$ with an edge at a given perturbative order $n_{0}$. The resummed behaviour at this point is easily obtained by noting that the first derivative $\hat{\sigma}^{\prime}(C)$ of the corresponding distribution is a safe quantity that has precisely a step at order $n_{0}$. Thus we can apply to $\hat{\sigma}^{\prime}(C)$ a DL resummation analogous to that carried out in Eq. (4.2), to obtain the following distribution

$$
\hat{\sigma}_{+}^{(\infty)}(C)=\hat{\sigma}_{-}^{(\infty)}\left(C_{0}\right)+\left(C-C_{0}\right) \hat{\sigma}_{-}^{(\infty) \prime}\left(C_{0}\right)+\left[\hat{\sigma}_{+}^{\left(n_{0}\right)^{\prime}}\left(C_{0}\right)-\hat{\sigma}_{-}^{\left(n_{0}\right)^{\prime}}\left(C_{0}\right)\right] \int_{0}^{C-C_{0}} d x e^{-\frac{a}{2} \ln ^{2} x}
$$

The first two terms on the right-hand side extrapolate $\hat{\sigma}_{-}^{(\infty)}(C)$ linearly into the region above the critical point $C=C_{0}$ and the last term bends this linear extrapolation smoothly, in proportion to the sharpness of the lower-order edge.

The resummation of double-sided singularities of the type encountered in jet profiles is technically more complicated. It leads to a smooth "jump" structure obtained by smearing (convoluting) two Sudakov shoulders: a shoulder above and an inverted shoulder below the critical point, or vice versa. As a result, the two sides of the lower-order step match with some intermediate value at the critical point in the all-order distribution and the step is smoothed. An example of this resummation carried out numerically for the jet shape $\psi(r)$ is presented in Ref. 24] (cf. Fig. 11). In general, the derivation of resummed expressions in analytic form is quite difficult and not always feasible. This is because the type of smearing (convolution) to be applied to the two shoulders is strongly dependent on the detailed kinematics. In the presence of double-sided singularities, the kinematics is usually very involved since it is responsible for the non-monotonic behaviour of the observable with respect to variations of particle angles and energies.

\section{Discussion and conclusions}

In this paper we have discussed in rather general terms the divergences which can arise inside the physical region in infrared- and collinear-safe quantities computed to any fixed order 
in perturbation theory. We have also given specific examples of widely-used quantities in $e^{+} e^{-}$and hadron-hadron physics for which these problems actually arise. The divergences correspond to integrable singularities and therefore they could in principle be removed by non-perturbative smearing effects. However, this would require non-perturbative contributions that are not power-suppressed at high energies.

We have argued that instead the remedy for these problems lies entirely within perturbation theory: the resummation of soft-gluon contributions to all orders should be sufficient to render any safe quantity finite and smooth throughout the physical phase space. Despite the violation of finiteness of fixed-order calculations, perturbative resummation suggests that non-perturbative effects are still power-suppressed in infrared- and collinear-safe observables.

A resummation of double-logarithmic terms was performed to illustrates how this works in the case of the $e^{+} e^{-} C$-parameter distribution (Fig. 1). More work remains to be done in order to resum single-logarithmic terms and the type of double-sided singularities encountered in hadronic-jet profiles.

The issues we have discussed are of some importance for QCD phenomenology. Before using any "safe" observable to test the theory or to measure $\alpha_{S}$, one needs to identify the critical points of that observable and the expected behaviour in whatever order of perturbation theory is to be used. These points will need to be avoided in comparisons between fixed-order predictions and experiment. On the other hand, if resummed predictions can be obtained to single-logarithmic precision, then the behaviour at critical points can be used to provide interesting new tests of QCD, as was the case for resummation near the exclusive phase-space boundary [4, 4 .

\section{Acknowledgments}

We are grateful for helpful discussions on this topic with M.H. Seymour, and for correspondence with W.T. Giele.

\section{References}

1. See, for instance: A. Bassetto, M. Ciafaloni and G. Marchesini, Phys. Rep. 100 (1983) 201; Yu.L. Dokshitzer, V.A. Khoze, A.H. Mueller and S.I. Troyan, Basics of Perturbative QCD (Editions Frontieres, Paris, 1991); R.K. Ellis, W.J. Stirling and B.R. Webber, QCD and Collider Physics (Cambridge Univ. Pr., Cambridge, 1996).

2. G. Sterman and S. Weinberg, Phys. Rev. Lett. 39 (1977) 1436.

3. J.C. Collins, D.E. Soper and G. Sterman, in Perturbative Quantum Chromodynamics, ed. A.H. Mueller (World Scientific, Singapore, 1989), p. 1 and references therein.

4. S. Catani, G. Turnock, B.R. Webber and L. Trentadue, Nucl. Phys. B407 (1993) 3. 
5. S. Catani, G. Turnock, B.R. Webber and L. Trentadue, Phys. Lett. 263B (1991) 491; S. Catani, G. Turnock, B.R. Webber, Phys. Lett. 272B (1991) 368, Phys. Lett. 295B (1992) 269.

6. J. Kodaira and L. Trentadue, Phys. Lett. 112B (1982) 66, Phys. Lett. 123B (1982) 335; C.T.H. Davies, W.J. Stirling and B.R. Webber, Nucl. Phys. B256 (1985) 413; J.C. Collins, D.E. Soper and G. Sterman, Nucl. Phys. B250 (1985) 199.

7. G. Sterman, Nucl. Phys. B281 (1987) 310; S. Catani and L. Trentadue, Nucl. Phys. B327 (1989) 323, Nucl. Phys. B353 (1991) 183.

8. S. Catani, G. Marchesini and B.R. Webber, Nucl. Phys. B349 (1991) 635.

9. E. Laenen, J. Smith and W.L. van Neerven, Nucl. Phys. B369 (1992) 543;

10. E.L. Berger and H. Contopanagos, Phys. Rev. D 54 (1996) 3085.

11. S. Catani, M.L. Mangano, P. Nason and L. Trentadue, Nucl. Phys. B478 (1996) 273.

12. G. Parisi, Phys. Lett. 74B (1978) 65;

J.F. Donoghue, F.E. Low and S.Y. Pi, Phys. Rev. D 20 (1979) 2759;

R.K. Ellis, D.A. Ross and A.E. Terrano, Nucl. Phys. B178 (1981) 421.

13. S. Catani and B.R. Webber, Cambridge preprint Cavendish-HEP-97/16, in preparation.

14. CDF Collaboration, F. Abe et al., Phys. Rev. D 70 (1993) 713; D0 Collaboration, S. Abachi et al., Phys. Lett. 357B (1995) 500.

15. E. Farhi, Phys. Rev. Lett. 39 (1977) 1587.

16. S. Catani and M.H. Seymour, Phys. Lett. 378B (1996) 287.

17. Z. Kunszt, P. Nason, G. Marchesini and B.R. Webber, in 'Z Physics at LEP 1', CERN 89-08, vol. 1, p. 373.

18. S.D. Ellis et al., in Proceedings of Research Directions for the Decade, Snowmass 1990, ed. E.L. Berger (World Scientific, Singapore, 1992), p. 134.

19. S. Catani, Yu.L. Dokshitzer, M.H. Seymour and B.R. Webber, Nucl. Phys. B406 (1993) 187.

20. S.D. Ellis and D.E. Soper, Phys. Rev. D 48 (1993) 3160.

21. UA2 Collaboration, J. Alitti et al., Phys. Lett. 257B (1991) 232.

22. CDF Collaboration, F. Abe et al., Phys. Rev. D 45 (1992) 1448;

D0 Collaboration, S. Abachi et al., Phys. Rev. D 53 (1996) 6000.

23. W.B. Kilgore and W.T. Giele, Phys. Rev. D 55 (1997) 7183;

W.B. Kilgore, preprint Fermilab-Conf-97/141-T (hep-ph/9705384).

24. M.H. Seymour, preprint RAL-97-026 (hep-ph/9707338). 
25. S.D. Ellis, Z. Kunszt and D.E. Soper, Phys. Rev. D 62 (1989) 726.

26. H. Baer, J. Ohnemus and J.F. Owens, Phys. Rev. D 40 (1989) 2844. 